\title{
Transcription factor YY1 expression in human gastrointestinal cancer cells
}

\author{
DHARMARAJ CHINNAPPAN ${ }^{1}$, DONGMEI XIAO ${ }^{1}$, ANITA RATNASARI $^{1}$, \\ CHRIS ANDRY ${ }^{2}$, THOMAS C. KING ${ }^{2}$ and H. CHRISTIAN WEBER ${ }^{1,2}$ \\ ${ }^{1}$ Section of Gastroenterology and ${ }^{2}$ Department of Pathology and Laboratory Medicine, \\ Boston University School of Medicine, Boston, MA, USA
}

Received January 5, 2009; Accepted February 16, 2009

DOI: 10.3892/ijo_00000270

\begin{abstract}
Over-expression of the multifunctional zinc-finger transcription factor Yin Yang 1 (YY1) has been associated with cellular proliferation and resistance to apoptotic stimuli. In this study, we report that YY1 was uniformly highly overexpressed in a wide range of human cancer cell lines and in human colon cancer tissue samples. The examination of YY1-specific mRNA expression demonstrated at least six mRNA isoforms ubiquitously expressed in normal human adult and fetal tissues. Substantial over-expression of two specific mRNA isoforms of 7.5 and $2.9 \mathrm{~kb}$ size, respectively, was detected in gastrointestinal and other cancer cells in vitro, whereby mRNA stability differed significantly between various cell lines. YY1 protein expression levels were similar in different colon cancer cell lines. Using FISH analysis of several colorectal cancer cell lines, the human YY1 locus was expectedly identified on chromosome $14 q 32$ and no evidence of gene amplification and chromosomal translocation was observed. However, varying degree of aneuploidy was noted in vitro. YY1 immunoreactivity in human colon tumor samples was found more intense in poorly differentiated tumors than in moderately and well differentiated colon cancers and lower expression levels tended to be associated with shorter survival. In conclusion, YY1 was over-expressed in colon cancer in the absence of gene amplification and chromosomal translocation. YY1 mRNA and protein stability are important regulatory mechanisms of YY1 expression in colon cancer.
\end{abstract}

Correspondence to: Dr H. Christian Weber, Section of Gastroenterology, Boston University School of Medicine, 650 Albany Street, EBRC, Room 515, Boston, MA 02118-2518, USA

E-mail: christian.weber@bmc.org

Abbreviations: FISH, fluorescent in situ hybridization; LOH, loss of heterozygosity; TP53, tumor suppressor protein p53; YY1, Ying Yang 1; MTN, multiple tissue Northern blot

Key words: YY1, FISH, RNA blot

\section{Introduction}

Malignancies of the colon represent one of the most common carcinomas in the Western world and its mortality rate ranks second only to lung cancer in the United States (1). Colon carcinogenesis is characterized by stepwise accumulation of several genetic and epigenetic changes during the transformation from normal colonic epithelial cells to frank carcinoma. Inactivating mutations of the tumor suppressor genes APC, DCC and TP53 and activating mutations of the oncogene K-ras were thought dominant during colorectal carcinogenesis (2-6). The more recent appreciation of microsatellite instability and the existence of additional transforming pathways demonstrated the heterogeneity of the molecular basis of colon cancer, including the $\mathrm{CpG}$ island methylation phenotype (CIMP) (6-11).

Along this vein, the multifunctional zinc-finger transcription factor YY1 has recently been assigned fundamental biological properties relevant to human malignancies. In prostate cancer tissue microarray samples YY1 nuclear and cytoplasmic expression was observed to be increased predominantly in neoplastic tissues and prostatic intraepithelial neoplasia (PIN) when compared to matched benign cells (12). In ERBB2-positive primary breast cancer samples an association of YY1, AP-2a and ERBB2 expression levels was reported (13) possibly due to ERBB2 promoter regulation by YY1 and AP-2 (14). Expression of YY1 was also reported in biopsy material from human osteosarcoma cells but not in benign osteoid tissue and was associated with tumor growth and invasiveness $(15,16)$. Furthermore, using an in silico approach, YY1 was identified as a commonly over-expressed gene in the majority of examined human epithelial cancers derived from prostate, breast and colon (17).

The human YY1 gene was mapped to chromosome 14q32, a locus that has been previously implicated in the pathogenesis of colorectal cancers (18-25) underscoring the possibility of a role of YY1 in colon tumor biology. The YY1 gene product has been found expressed in a variety of different mammalian cell lines and it has been proposed that YY1 is a ubiquitously expressed GLI-Krüppel class zinc finger transcription factor in normal human and mouse tissues (26). However, original experimental data supporting the latter notion have not been published to our knowledge. Prompted by the possible link of 
YY1 and colon cancer as well as the paucity of knowledge regarding its normal tissue distribution, we determined YY1 expression in normal mouse, human adult and fetal tissues as well as in a series of human cancer cell lines, characterized its mRNA isoforms and demonstrated its significant overexpression in human colon cancer samples.

\section{Materials and methods}

Materials. Antibodies for YY1 were from Santa Cruz (sc-7341; Santa Cruz, CA); antibodies against $\beta$-actin were purchased from Sigma (St. Louis, MO). Actinomycin D and other chemicals were from Sigma unless stated otherwise.

Cell culture. A431, LoVo, Caco2, HT-29, DLD-1 cells were obtained from the American Type Culture Collection (Manassas, VA) and maintained in minimum essential medium Eagle (MEM) supplemented with $10 \%$ fetal calf serum at $37^{\circ} \mathrm{C}$ in $5 \% \mathrm{CO}_{2}$ atmosphere.

Western blot analysis. Cells were harvested and lysed in ice-cold lysis buffer $(0.15 \mathrm{M}$ sodium chloride, $50 \mathrm{mM}$ Tris, $0.5 \%$ Triton $\mathrm{X}-100$ and protease inhibitors cocktail from Roche, Indianapolis, IN) for $30 \mathrm{~min}$, followed by centrifugation at 14,000 rpm for $10 \mathrm{~min}$ and the pellet was discarded. Protein concentration of the cell lysate was detected by protein assay kit from Bio-Rad (Hercules, CA) and adjusted such that each sample contained an equal amount of protein. Protein samples were dissolved in loading buffer $(60 \mathrm{mM}$ Tris- $\mathrm{HCl}, \mathrm{pH}$ 6.8, 2\% SDS, $100 \mathrm{mM}$ dithiothreitol, and $0.01 \%$ bromophenol blue); heated to $100^{\circ} \mathrm{C}$ for $5 \mathrm{~min}$, and loaded onto the gel in electrophoresis buffer containing $25 \mathrm{mM}$ Tris- $\mathrm{HCl}, \mathrm{pH} 8.3,250 \mathrm{mM}$ glycine, and 0.1\% SDS. After the completion of electrophoresis, proteins were transferred to a nitrocellulose membrane (Hybond ECL, Amersham Life Science). Membranes were blocked with 5\% non-fat powdered milk in TBS (10 mM Tris, pH 7.5, $100 \mathrm{mM} \mathrm{NaCl}$, $0.1 \%$ Tween-20) and incubated with the YY1 primary antibody (Santa Cruz, sc-7341) at $4^{\circ} \mathrm{C}$ overnight followed by horseradish peroxidase-conjugated secondary antibody. Immuno-complexes were visualized using the ECL Western blotting detection reagents (Amersham Pharmacia Biotech, Piscataway, NJ).

Northern blot analysis. Total cellular RNA was isolated using the RNeasy kit from Qiagen (Valencia, CA) or TRIzol reagent (Invitrogen Corp.; Carlsbad, CA) according to the recommendations by the manufacturer. Aliquots of $20 \mu \mathrm{g}$ of total cellular RNA were denatured in gel-running buffer [0.04 M 3-(N-morpholino)propanesulfonic acid/10 mM sodium acetate/0.5 mM EDTA, $\mathrm{pH} 7.5 / 50 \%$ formamide/6\% formaldehyde] and then electrophoresed on a 1\% agarose/6\% formaldehyde gel. The integrity of the RNA samples was determined by the visualization of $28 \mathrm{~S}$ and $18 \mathrm{~S}$ ribosomal RNA bands with ethidium bromide staining $(10 \mu \mathrm{g} / \mathrm{ml})$. After electrophoresis at $10 \mathrm{~V} / \mathrm{cm}$, the RNA was transferred from the gel to a nylon filter (Schleicher \& Schuell, Keene, $\mathrm{NH}$ ) by capillary action, as described by Thomas (27). Multiple tissue Northern blots (Clontech, Palo Alto, CA) were used to determine the YY1 expression in human and mouse tissues by hybridization with the radioactively labeled full-length human YY1 cDNA.

Densitometry. Autoradiograms of Northern blot analyses were scanned and intensities of bands were determined using the Model GS-700 Imaging Densitometer (Bio-Rad, Hercules, CA). Results are expressed as arbitrary units whereby the corresponding human $B$-actin mRNA expression levels were used to normalize YY1 mRNA isoform expression levels. Background levels were determined in representative areas on each autoradiogram, averaged and subtracted from each individual densitometry reading.

Cytogenetics and fluoresence in situ hybridization (FiSH). Cytogenetic studies were performed as described elsewhere (28). The BAC clone containing the YY1 gene (RP11-3621.22) was purchased and DNA was extracted by using a large construct kit (Qiagen). The YY1 probe was directly labeled with spectrum green dUTP (Vysis Inc., IL). The sub-telomeric probe for the human chromosome 14 was purchased from Vysis (Downers Grove, IL). FISH technique was carried out by standard procedure (28) and analyses were performed using Nikon fluorescent microscope with a CCD camera.

Immunohistochemistry. Two identical human tissue microarrays were purchased from Imgenex, Inc. (cat\# IMH-359, San Diego, CA). Tissue microarrays containing 60 characterized colonic tissue cores $(2 \mathrm{~mm})$, including one normal sample, were de-paraffinized and subjected to antigen retrieval using low $\mathrm{pH}$ citrate buffer with microwave treatment. Slides were incubated with a monoclonal antibody specific for human YY1 (H-10; Santa Cruz, sc-7341) for one hour at room temperature. After washing, signal was detected using a Multilink HRP kit (Biogenex) with DAB as the chromagen. Internal controls gave appropriate results with specific nuclear staining. The tissue sections were scored for nuclear staining intensity $(0$, no staining to 6 , intense staining) in tumor cells and non-neoplastic colonocytes.

\section{Results}

Expression of YY1 mRNA in normal human tissue. While it has been reported that YY1 is a ubiquitously expressed gene, data on expression pattern in normal human and mouse tissues are surprisingly absent in the literature. Therefore, we first performed hybridization experiments of several multiple tissue mRNA blots (Clontech) representing a wide array of normal human and mouse tissues. Using a full-length human YY1 cDNA probe, we detected human and mouse YY1 mRNA expression present in all samples tested. Very low expression levels were noted in the human lung, colon and small intestine, whereas in contrast, skeletal muscle and heart displayed by far the most abundant expression levels (Fig. 1). Furthermore, we identified at least six YY1 mRNA isoforms present in all tissue samples with the most prominent ones of approximately 4.9, 2.9 and $1.2 \mathrm{~kb}$ in length (Fig. 1A). Weak bands of approximately $0.5,1.9$ and $7.5 \mathrm{~kb}$ were also present in adult human tissues. The expression pattern and levels of human YY1 mRNA isoforms in fetal tissues, including lung, brain, kidney and liver (MTN F; Clontech) 

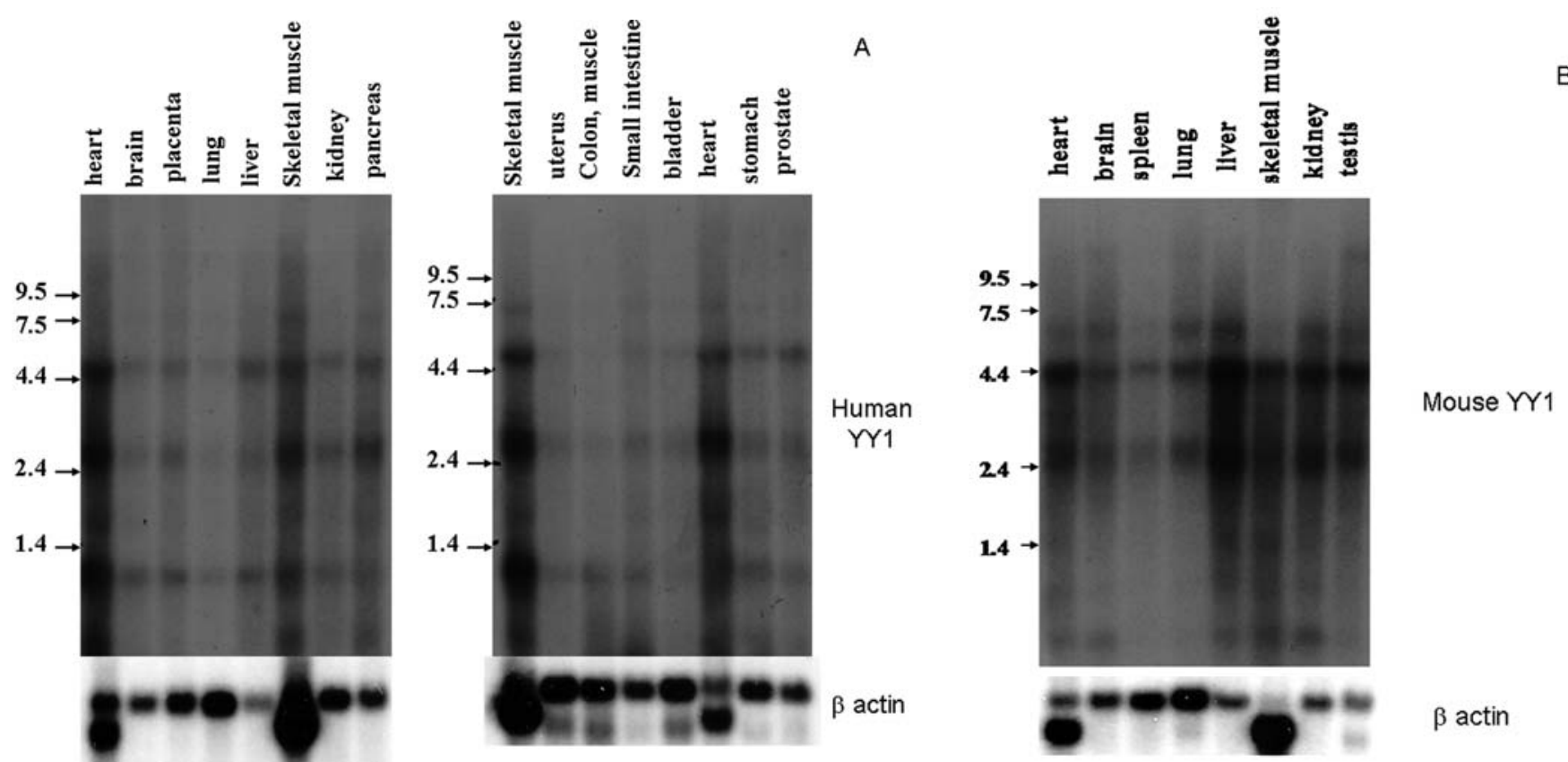

Figure 1. Human and mouse YY1 mRNA expression in normal tissues samples is ubiquitous. Human and mouse multiple mRNA tissue blots were hybridized first with the radioactively labeled full-length human YY1 cDNA, washed and exposed to X-ray film as described in Materials and methods. Nylon filters were then stripped and re-probed with a human B-actin probe. A displays two MTN (tissues as indicated) showing the human YY1 multiple specific mRNA isoforms with most abundant expression in normal human heart and skeletal muscle. The autoradiogram of the mouse MTN is shown in B.

were similar to those detected in adult tissues (data not shown). At least six mouse mRNA isoforms were distinguished, including the most abundantly expressed of approximately 2.6, 4.3 and $6.5 \mathrm{~kb}$ in size (Fig. 1B).

YY1 mRNA isoform over-expression in human cancer cell lines. Prompted by previous reports of YY1 over-expression in human cell lines and our findings in normal human tissues, we then determined YY1 mRNA expression in a series of human cancer cells and one mouse colorectal cancer cell line (MC-26) using identical amounts of total cellular RNA of each cell line in Northern blot analyses. Cell lines that were examined included colon adenocarcinoma cells (HT-29, DLD-1, Caco-2), gastric cancer SIIA cells, a pancreatic endocrine cancer cell line BON-I, epidermoid carcinoma A431 cells and HeLa cervical cancer cells. The autoradiogram and corresponding densitometry analyses are shown in Fig. 2. When compared with the normal human tissue of highest YY1 mRNA expression level, the skeletal muscle, all tested cell lines demonstrated over-expression YY1 mRNA isoforms 7.5 and $2.9 \mathrm{~kb}$. Densitometry data (Fig. 2B) were obtained from the autoradiogram (Fig. 2A) and all data points were normalized to human $B$-actin expression level. The major YY1 mRNA $2.9 \mathrm{~kb}$ isoform was found 3-6-fold over-expressed in cancer cells, except in gastric cancer SIIA cells with similar levels as compared to skeletal muscle. Correspondingly, the YY1 mRNA 7.5-kb isoform over-expression was detected to be manifold higher and ranged from 2-3-fold in BON-I cells to 40-60-fold in A431 and HeLa cells, respectively (Fig. 2B). Moreover, when Northern blots were exposed for various time periods, an apparent loss of two normal human YY1 mRNA isoforms of approximately 4.9 and $1.9 \mathrm{~kb}$, was noted in HT-29, Caco-2 and DLD-1 CRC cells (Fig. 2C; asterisk).

YY1 mRNA stability. Based on the absence of evidence for YY1 gene amplification and translocation, we began to examine human YY1 mRNA stability in selected cancer cell lines. We chose two colon cancer cell lines with extensive aneuploidy (Caco-2) and almost normal karyotype (LoVo-1) as well as A431 cells. Cells were maintained in the presence of actinomycin D $(10 \mu \mathrm{g} / \mathrm{ml})$ to inhibit RNA synthesis for a time period as indicated, total cytoplasmic RNA was isolated and then subjected to Northern blot analysis (Fig. 3, top panel). Both YY1 mRNA isoforms of 2.9 and $7.5 \mathrm{~kb}$ were depicted and densitometry analysis was performed with normalization to human B-actin expression levels (Fig. 3, bottom panel). Estimation of $2.9 \mathrm{~kb}$ mRNA half-lives in three different cell lines (LoVo $\sim 3 \mathrm{~h}, \mathrm{~A} 431 \sim 4 \mathrm{~h}$, and Caco-2 8 h) suggested varying mRNA stability. The less abundant $7.5 \mathrm{~kb}$ mRNA isoform showed similar stability when compared to the corresponding $2.9 \mathrm{~kb}$ isoform in the same cell line (Fig. 3, bottom panel). Additional experiments in several colon cancer cells showed cell line specific YY1 mRNA stability of considerable variation while YY1 protein expression levels were very similar (data not shown).

YY1 chromosomal localization and exclusion of gene amplification. We performed FISH analysis on a selected number of colon cancer and A431 cells to examine the possibility of gene amplification and chromosomal translocation. Five colorectal cancer cell lines, A431 cells and peripheral white blood cells, serving as a control sample, 


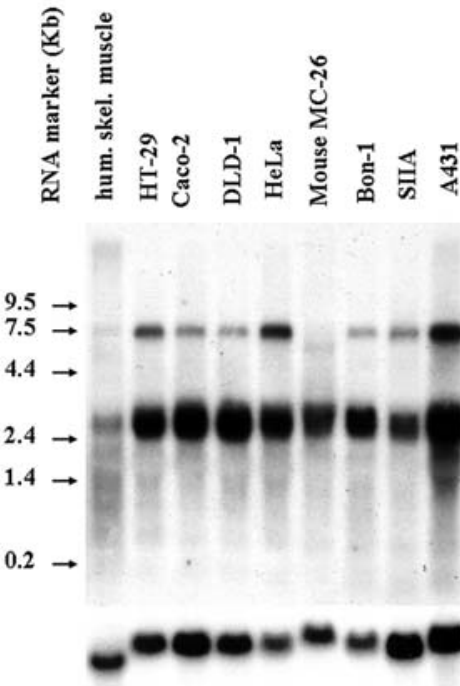

Figure 2. Over-expression and loss of human YY1 mRNA isoforms in gastrointestinal cancer cells. (A) Twenty $\mu \mathrm{g}$ of total cellular RNA from normal heart and human skeletal muscle and various human cancer cell lines were subjected to Northern blot analysis as indicated. The nylon filter was hybridized with radioactively labeled, full-length human YY1 cDNA, washed at high stringency and exposed to X-ray film (top panel). The filter was then stripped and re-probed with a human B-actin probe shown in the bottom panel. As compared with the human RNA from normal skeletal muscle (leftmost lane), all cancer cell lines showed evidence of significant over-expression of YY1 mRNA isoforms 7.5 and $2.9 \mathrm{~kb}$. (B) The corresponding quantitative densitometry analysis of YY1 mRNA isoforms 2.9 and $7.5 \mathrm{~kb}$ is depicted in B. C shows the loss of two YY1 mRNA isoforms 4.9 and $1.9 \mathrm{~kb}$ (asterisk) in CRC cell lines when compared with the normal human YY1 mRNA pattern.
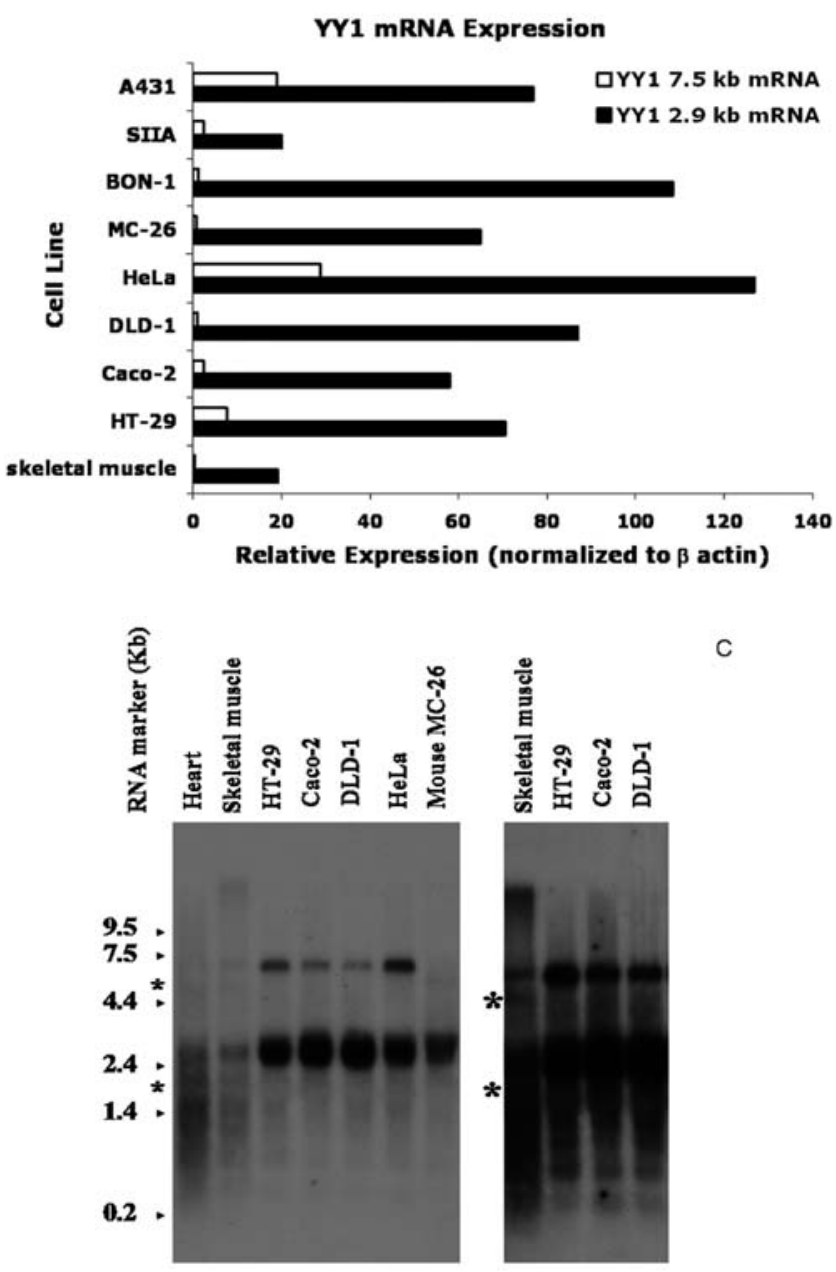
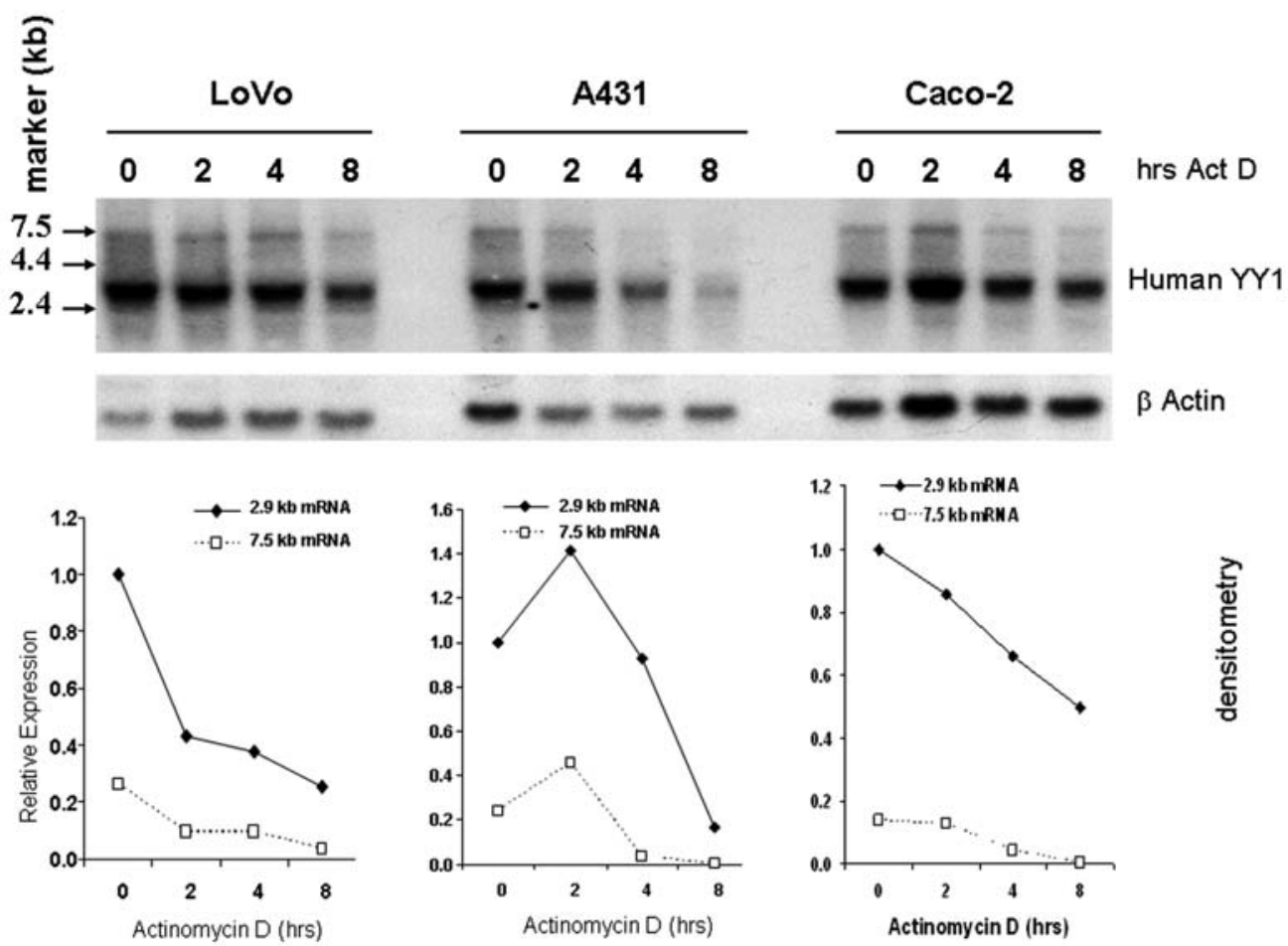

$\beta$ Actin

Figure 3. YY1 mRNA stability varies in human cancer cells. Top panel, human cancer cells were grown in culture and exposed to actinomycin D (10 $\mu \mathrm{g} / \mathrm{ml})$ for indicated times, total cellular RNA was isolated, subjected to Northern blot analysis and hybridized with radioactively labeled full-length human YY1 cDNA and human B-actin as described. Bottom panel, the densitometry analysis of the autoradiogram is shown. Expression levels are depicted as relative values (YY1/B-actin), whereby the control value at time 0 was arbitrarily set as 1 . Human YY1 mRNA stability varies significantly in cell lines examined. 

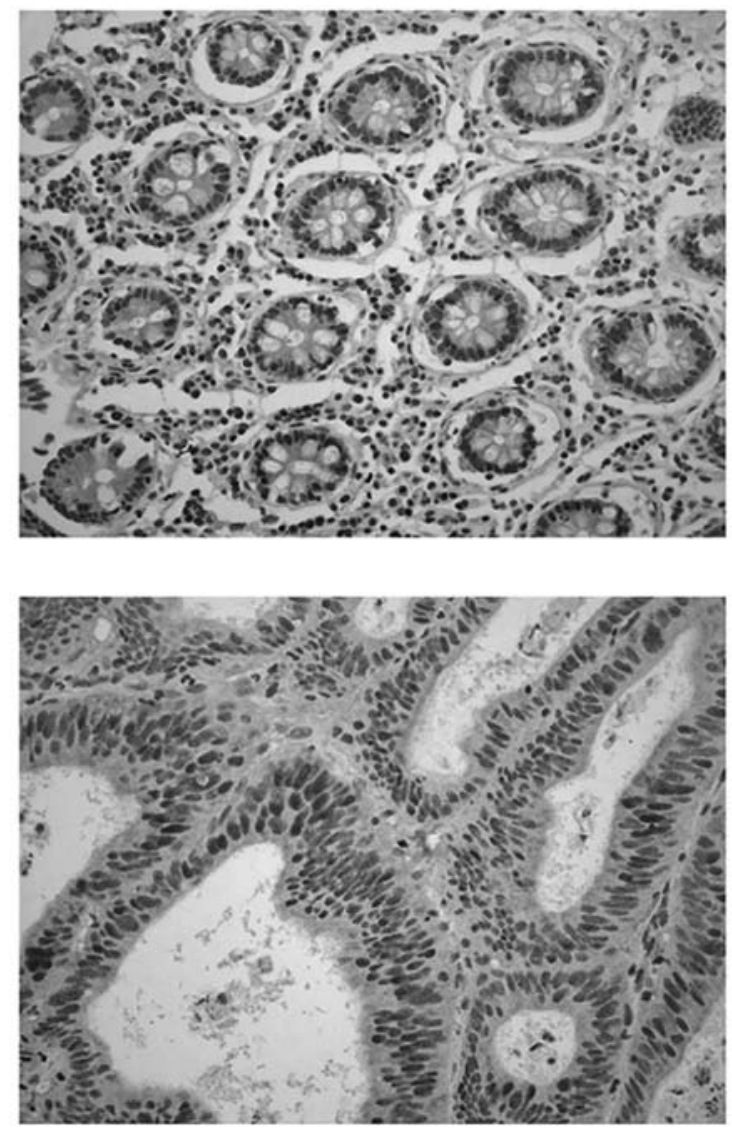

Figure 4. Expression of human YY1 in tissue samples of normal colon and colorectal cancer specimens. Immunohistochemistry was performed on tissue array specimens of colorectal cancer using an anti-human YY1 antibody. The top panel (A) shows a normal control sample (magnification $\mathrm{x} 200$ ) and (B) depicts an adenocarcinoma of the colon (magnification $\mathrm{x} 400$ ).

were included. We used two sets of probes, one specifically labeling theYY1 gene in green color and the other one was the sub-telomeric probe for chromosome 14 red in color. As expected, peripheral blood cells showed a normal karyotype and FISH demonstrated two signals in each nucleus for the YY1 gene (not shown). We determined that in almost all interphase cells, the number of YY1-specific green signals was exactly equal to the number of sub-telomeric red signals mapping to chromosome 14. As previously reported, we mapped the human YY1 gene to chromosome band 14q32. Moreover, all five colorectal cancer cell lines and A431 cells displayed various degrees of aneuploidy by YY1-specific FISH, which correlated well with their numerically abnormal karyotypes (Table I). The colon cancer cells Caco-2 and epidermoid A431 cancer cells appeared to possess the most abnormal findings. In HT-29 cells $87 \%$ cells were observed with two YY1 signals but karyotypically this cell line is monosome for chromosome 14. Importantly, these FISH experiments also showed the absence of gene amplification and chromosomal translocations of the human YY1 locus, thus suggesting strongly that other molecular mechanisms are likely to be relevant in YY1 over-expression in human cancer.

YY1 protein expression in cancer cells. Protein YY1 expression was determined in four colon carcinoma cell lines (Caco-2,
Table I. Summary of results from molecular cytogenetic studies in human cancer cells with FISH for YY1.

No. of signals detected in interphase chromosomes

\begin{tabular}{lcrrrr}
\cline { 2 - 6 } Cell line & 1 & 2 & 3 & 4 & $\geq 5$ \\
\hline Control & & 100 & & & \\
LoVo & & 95 & 4 & 1 & \\
A431 & & 15 & 46 & 31 & 9 \\
HT-29 & 3 & 87 & 3 & 7 & \\
DLD-1 & 21 & 70 & 3 & 6 & \\
Caco-2 & & 5 & 36 & 32 & 27 \\
\hline
\end{tabular}

FISH was performed in cells as indicated using a probe for the human YY1 gene. One hundred interphase chromosomes were analyzed quantitatively. Numbers represent the percent of signals detected in preparations.

DLD-1, HT-29, and LoVo-1) and in A431 carcinoma cells. Expression levels in all colon carcinoma cell lines were almost identical whereas YY1 expression in A431 cells was found to be substantially lower (data not shown). Protein stability in A431, Caco-2 and LoVo-1 cells was determined by treatment with cycloheximide $(10 \mu \mathrm{g} / \mathrm{ml})$. YY1 protein levels remained stable in A431 and Caco-2 cells without apparent decay for $6 \mathrm{~h}$ whereas YY1 levels in LoVo-1 cells diminished by $50 \%$ after $6 \mathrm{~h}$ in the presence of cycloheximide treatment (data not shown). A study by Austen et al, in various mammalian cell lines and YY1 protein half-life was determined as 3.5-4 h similar in each cell line examined (29). Our own studies suggested that YY1 half-life in LoVo-1 cells is comparable to the previous study ( $3 \mathrm{~h}$ ) whereas in A431 and Caco-2 cells YY1 protein was found considerably more stable (data not shown).

YY1 expression in colon cancer tissue. To further explore the expression of YY1 in colon cancer, we performed immunohistochemistry (IHC) studies in colon cancer tissue microarrays. Two identical microarrays were subjected to IHC using a YY1-specific monoclonal antibody. YY1 expression was exclusively detected in nuclei but not in cytoplasm and no staining was seen in mitotic cells. YY1 immunostaining was detectable in normal colon epithelial cells and colon cancer cells as well as lymphocytes, neutrophils and muscle cells. Although our sample size $(n=45)$ was too small for a sufficiently powered statistical analysis, score values of YY1 staining showed a trend toward increased staining in poorly differentiated and mucinous tumors (median scores 92 and 135, respectively) compared with well and moderately differentiated tumors (median scores 60 and 80). In addition, slightly increased staining was observed in tumors from females (median score 80) when compared to those from male patients (median score $65)$. When samples were dichotomized to tumors with score values of $<60$ and $\geq 60$, the hazard ratio of survival for the former was 2.0, however, these comparisons were not statistically significant. Representative examples of IHC of 
a normal core and a colon adenocarcinoma are depicted in Fig. 4.

\section{Discussion}

This present study is the first to provide evidence of YY1 over-expression in a series of human colon cancer samples. While it has been reported that YY1 is a ubiquitously expressed gene, experimental data were narrowly confined to few permanent human and rodent tumor cell lines. Predictions were mainly based on data mining using AceView $(26,29,30)$. This present report is the first to characterize YY1 specific transcripts in normal human and mouse tissues (Fig. 1). Our expression studies of normal human and mouse tissues characterized at least 6 distinct YY1 mRNA isoforms in both species (Fig. 1) and these findings are in line with predictions provided by AceView (30). However, one additional abundant YY1 mRNA of approximately $4.9 \mathrm{~kb}$ was consistently identified in all normal human tissues. In contrast, when a series of human cancer cells was examined by Northern blot analysis, YY1 specific mRNAs were characterized by robust over-expression of isoforms 2.9 and $7.5 \mathrm{~kb}$ and the absence of 4.9 and $1.9 \mathrm{~kb}$ (Fig. 2) isoforms. It is likely of considerable significance that these specific mRNA isoforms remained undetectable in cancer cells in vitro (Fig. 2C) and the biological relevance of these findings remains to be fully determined in future studies. The specific YY1 mRNA pattern of gastrointestinal cancer cells (Fig. 2) was found identical in other cancer cells derived from prostate, melanoma and breast (data not shown).

Although a rare event in colon cancer (31), we also excluded the possibility of YY1 gene amplification and chromosomal translocation as underlying mechanisms of its over-expression in several colon cancer cell lines by FISH analysis (Table I). This suggests that chromosomal instability characterized by aneuploidy represents a major pathological event in colon cancer cells. Similar findings were reported previously in a comprehensive genomic survey of colon cancer cell lines examined by CGH, G-banding and M-FISH which noted no recurrent chromosomal translocations. The authors emphasized the similarities of their findings in cancer cell lines in vitro with those detected in primary colon cancers suggesting that numerical and structural abnormalities of these cell lines were representative of colon tumors in vivo (32). Furthermore, some previous genome-wide studies of colon cancer samples determined a significant prevalence of loss of heterozygosity (LOH) at chromosome $14 \mathrm{q}$, specifically in tissues from early age onset colorectal cancer patients, metastatic late stage disease and recurrent metastatic disease (18-25). This would imply that YY1 might be inactivated and could be a candidate tumor suppressor gene.

Finally, YY1 protein was observed increased in nuclear and cytoplasmic expression in osteosarcomas compared to normal bone tissue suggesting a possible role in osteoblastic transformation (15). The precise mechanisms of YY1 overexpression in epithelial tumors and their specific molecular consequences still remain to be determined although YY1 has been reported to affect cellular pathways and checkpoints critical in tumor biology including histone modification, cell cycle control, cell proliferation and apoptosis (14,26,33-42).
Further studies with larger sample size cohorts of colon tumors and the analysis of their respective clinical outcomes will determine whether YY1 expression levels might be useful as a molecular signature in colon cancer. While our sample size of colon cancer cores on a tissue microarray was relatively small and statistical analysis did not reach significant levels, YY1 immunoreactivity of malignant colonocytes was observed exclusively in the nuclear compartments, did not stain mitotic cells. It was more intense in high grade colon tumors and survival tended to be poorer with lower YY1 immunoreactivity scores. Only a very limited number of studies regarding YY1 expression in human cancers in vivo have been published $(12,13,15)$. One study in prostate cancer determined predominant cytoplasmic and nuclear YY1 over-expression in neoplastic cells of intermediate to high morphological grade cancers and PIN. Our experimental results overall suggest that YY1 over-expression in colon cancer cells might occur due to various complex mechanisms, including transcriptional, post-transcriptional and post-translational regulation as well as alternate promoter use.

\section{Acknowledgements}

We thank Dr S. Georas (John's Hopkins University, Baltimore, MD) for the YY1 plasmid constructs and Dr Courtney M. Townsend Jr (UT Medical Branch, Galveston, TX) for SIIA and Bon-I cells. Financial support was provided in part by NIH (DK02722, DK063209) to H.C.W.

\section{References}

1. Jemal A, Siegel R, Ward E, et al: Cancer statistics, 2008. CA Cancer J Clin 58: 71-96, 2008.

2. Fearon ER, Cho KR, Nigro JM, et al: Identification of a chromosome $18 \mathrm{q}$ gene that is altered in colorectal cancers. Science 247: 49-56, 1990.

3. Fearon ER and Vogelstein B: A genetic model for colorectal tumorigenesis. Cell 61: 759-767, 1990.

4. Baker SJ, Fearon ER, Nigro JM, et al: Chromosome 17 deletions and p53 gene mutations in colorectal carcinomas. Science 244: 217-221, 1989 .

5. Vogelstein B, Fearon ER, Hamilton SR, et al: Genetic alterations during colorectal-tumor development. N Engl J Med 319: 525-532, 1988.

6. Segditsas $\mathrm{S}$ and Tomlinson I: Colorectal cancer and genetic alterations in the Wnt pathway. Oncogene 25: 7531-7537, 2006.

7. O'Brien MJ: Hyperplastic and serrated polyps of the colorectum. Gastroenterol Clin North Am 36: 947-968, 2007.

8. Jass JR: Classification of colorectal cancer based on correlation of clinical, morphological and molecular features. Histopathology 50: 113-130, 2007.

9. Chan $\mathrm{AO}$ and Rashid $\mathrm{A}: \mathrm{CpG}$ island methylation in precursors of gastrointestinal malignancies. Curr Mol Med 6: 401-408, 2006.

10. Kondo Y and Issa JP: Epigenetic changes in colorectal cancer. Cancer Metastasis Rev 23: 29-39, 2004.

11. Lengauer C, Kinzler KW and Vogelstein B: Genetic instability in colorectal cancers. Nature 386: 623-627, 1997.

12. Seligson D, Horvath S, Huerta-Yepez S, et al: Expression of transcription factor Yin Yang 1 in prostate cancer. Int J Oncol 27: 131-141, 2005.

13. Allouche A, Nolens G, Tancredi A, et al: The combined immunodetection of AP-2 alpha and YY1 transcription factors is associated with ERBB2 gene overexpression in primary breast tumours. Breast Cancer Res 10: R9, 2008.

14. Begon DY, Delacroix L, Vernimmen D, Jackers P and Winkler R: Yin Yang 1 cooperates with activator protein 2 to stimulate ERBB2 gene expression in mammary cancer cells. J Biol Chem 280: 24428-24434, 2005. 
15. De Nigris F, Botti C, De Chiara A, et al: Expression of transcription factor Yin Yang 1 in human osteosarcomas. Eur J Cancer 42: 2420-2424, 2006.

16. De Nigris F, Rossiello R, Schiano C, et al: Deletion of Yin Yang 1 protein in osteosarcoma cells on cell invasion and CXCR4/angiogenesis and metastasis. Cancer Res 68: 1797-1808, 2008.

17. Pilarsky C, Wenzig M, Specht T, Saeger HD and Grutzmann R: Identification and validation of commonly overexpressed genes in solid tumors by comparison of microarray data. Neoplasia 6 : 744-750, 2004.

18. Ookawa K, Sakamoto M, Hirohashi S, et al: Concordant p53 and DCC alterations and allelic losses on chromosomes $13 \mathrm{q}$ and $14 \mathrm{q}$ associated with liver metastases of colorectal carcinoma. Int J Cancer 53: 382-387, 1993.

19. Sasaki M, Okamoto M, Sato C, et al: Loss of constitutional heterozygosity in colorectal tumors from patients with familial polyposis coli and those with non-polyposis colorectal carcinoma. Cancer Res 49: 4402-4406, 1989.

20. Al-Mulla F, Behbehani AI, Bitar MS, Varadharaj G and Going JJ: Genetic profiling of stage I and II colorectal cancer may predict metastatic relapse. Mod Pathol 19: 648-658, 2006.

21. Al-Mulla F, AlFadhli S, Al-Hakim AH, Going JJ and Bitar MS Metastatic recurrence of early-stage colorectal cancer is linked to loss of heterozygosity on chromosomes 4 and $14 \mathrm{q}$. J Clin Pathol 59: 624-630, 2006.

22. Dai YC, Ho CL, Tsai YC, et al: Allelic loss of $14 \mathrm{q} 32$ in the pathogenesis of gastrointestinal and ampullary malignancies: mapping of the target region to a 17-cm interval. J Cancer Res Clin Oncol 131: 94-100, 2005.

23. Weber TK, Conroy J, Keitz B, et al: Genome-wide allelotyping indicates increased loss of heterozygosity on $9 p$ and $14 q$ in early age of onset colorectal cancer. Cytogenet Cell Genet 86: 142-147, 1999.

24. Young J, Leggett B, Ward M, et al: Frequent loss of heterozygosity on chromosome 14 occurs in advanced colorectal carcinomas. Oncogene 8: 671-675, 1993.

25. Diep CB, Kleivi K, Ribeiro FR, Teixeira MR, Lindgjaerde OC and Lothe RA: The order of genetic events associated with colorectal cancer progression inferred from meta-analysis of copy number changes. Genes Chromosomes Cancer 45: 31-41, 2006.

26. Shi Y, Lee JS and Galvin KM: Everything you have ever wanted to know about Yin Yang 1. Biochim Biophys Acta 1332: F49-F66, 1997.

27. Thomas PS: Hybridization of denatured RNA and small DNA fragments transferred to nitrocellulose. Proc Natl Acad Sci USA 77: 5201-5205, 1980 .
28. Chinnappan D, Zhang Y and Ravid K: AIM-1 transgenic mice with a curly tail phenotype and its chromosome location. Cytogenet Genome Res 98: 231A, 2002.

29. Austen M, Cerni C, Luscher-Firzlaff JM and Luscher B: YY1 can inhibit $\mathrm{c}-\mathrm{Myc}$ function through a mechanism requiring DNA binding of YY1 but neither its transactivation domain nor direct interaction with c-Myc. Oncogene 17: 511-520, 1998.

30. Thierry-Mieg D and Thierry-Mieg J: AceView: a comprehensive cDNA-supported gene and transcripts annotation. Genome Biol 7 (Suppl. 1): S1211-1214, 2006.

31. Croce CM: Oncogenes and cancer. N Engl J Med 358: 502-511, 2008.

32. Kleivi K, Teixeira MR, Eknaes M, et al: Genome signatures of colon carcinoma cell lines. Cancer Genet Cytogenet 155: 119-131, 2004

33. Gordon S, Akopyan G, Garban H and Bonavida B: Transcription factor YY1: structure, function, and therapeutic implications in cancer biology. Oncogene 25: 1125-1142, 2006.

34. Krippner-Heidenreich A, Walsemann G, Beyrouthy MJ, et al: Caspase-dependent regulation and subcellular redistribution of the transcriptional modulator YY1 during apoptosis. Mol Cell Biol 25: 3704-3714, 2005.

35. Palko L, Bass HW, Beyrouthy MJ and Hurt MM: The Yin Yang-1 (YY1) protein undergoes a DNA-replication-associated switch in localization from the cytoplasm to the nucleus at the onset of S phase. J Cell Sci 117: 465-476, 2004

36. Petkova V, Romanowski MJ, Sulijoadikusumo I, et al: Interaction between YY1 and the retinoblastoma protein. Regulation of cell cycle progression in differentiated cells. J Biol Chem 276: 7932-7936, 2001.

37. Rezai-Zadeh N, Zhang X, Namour F, et al: Targeted recruitment of a histone H4-specific methyltransferase by the transcription factor YY1. Genes Dev 17: 1019-1029, 2003.

38. Sui G, Affar el B, Shi Y, et al: Yin Yang 1 is a negative regulator of p53. Cell 117: 859-872, 2004.

39. Thomas MJ and Seto E: Unlocking the mechanisms of transcription factor YY1: are chromatin modifying enzymes the key? Gene 236: 197-208, 1999.

40. Wu S, Murai S, Kataoka K and Miyagishi M: Yin Yang 1 induces transcriptional activity of p73 through cooperation with E2F1. Biochem Biophys Res Commun 365: 75-81, 2008.

41. Yakovleva T, Kolesnikova L, Vukojevic V, et al: YY1 binding to a subset of p53 DNA-target sites regulates p53-dependent transcription. Biochem Biophys Res Commun 318: 615-624, 2004.

42. Yao YL, Yang WM and Seto E: Regulation of transcription factor YY1 by acetylation and deacetylation. Mol Cell Biol 21: 5979-5991, 2001 